\title{
Velocity-Range Relation for Fission Fragments
}

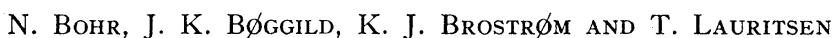 \\ Institute for Theoretical Physics, University of Copenhagen, Copenhagen, Denmark
}

(Received September 3, 1940

$I^{1}$ $\mathrm{N}$ two recent notes ${ }^{1,2}$ a number of features of the penetration of uranium fission fragments through matter as revealed by cloud-chamber photographs were discussed. In particular, it was pointed out that we have here to do with a velocity-range relation of a novel character which depends on the interplay between electron capture by the fragments over their entire range, and the ultimate stopping by nuclear collisions. It was also shown in this connection that the rapid fall of the total charge of the fragments with decreasing velocity implies that even a considerable difference in mass and charge of the nuclear fragments will have a relatively small effect on the total range of fragments emitted with the same momentum. Because of this fact, the preliminary study of the cloud-chamber tracks did not immediately indicate the presence of the two main groups of fragments which are shown so clearly in the chemical analysis of the fission products as well as in measurements of the total ionization produced by individual fragments. ${ }^{3} \mathrm{~A}$ closer analysis of a large new body of material, however, gives definite evidence of the presence of two groups of tracks and yields at the same time some further information regarding the range-velocity relation.

This evidence is obtained partly from the measurement of the total ranges of a small number of selected tracks from thin uranium layers, and partly from the counting of branches at various parts of the range of a large number of tracks originating in thick uranium targets. The range measurements of twelve tracks, of which eight were members of pairs ejected in opposite directions and four were single tracks, yield two broad groups with 22 - and 29 -cm range in normal air. Because of the rather large straggling within each group, it is difficult, however, to draw quite certain conclusions on the basis of so few measurements. It is therefore fortunate

${ }^{1}$ K. J. Bostr $\phi \mathrm{m}$, J. K. B $\varnothing$ ggild and T. Lauritsen, Phys. Rev. 58, 651 (1940).

${ }^{2}$ N. Bohr, Phys. Rev. 58, 654 (1940)

${ }^{3}$ Cf. L. Turner, Rev. Mod. Phys. 12, 22 (1940). that it is possible to obtain from a statistical analysis of the number of branches which are such a conspicuous feature of the tracks, still stronger evidence for the grouping. Because of the entirely fortuitous distribution of the branches, it is, of course, not possible to make any such separation on the basis of the branching of individual tracks, but, from the way in which the number of branches in a given portion of the range are distributed for a large number of tracks the grouping is quite evident.

If the probability for branching were the same for all tracks at a given distance from the end of the range, the number of branches with lengths within given limits in a certain portion of the track should be distributed according to the well-known law ${ }^{4}$

$$
P(n)=\omega^{n} e^{-\omega} / n !,
$$

where $\omega$ is the mean value of the number of branches in the interval considered and $P(n)$ is the expectation of the occurrence of just $(n)$ branches in this interval. Actual measurements on several hundred tracks in argon gas gives the result that in the region of the track from 0.3 to $1.15 \mathrm{~cm}$ (normal air) from the end of the range, the mean value of the number of branches is about 2.2 and the proportion of tracks which have $0,1,2$ and 3 branches are, respectively, $0.17,0.25,0.20$ and 0.15 , while the formula gives $0.11,0.24,0.27$ and 0.20 . From these figures, it is quite clear that the probability of branching for the different tracks is not the same. An almost perfect fit to the experimental values is obtained, however, if we assume that the tracks fall into two groups differing in their mean numbers of branches by a factor of three. A similar analysis of the branch distribution in the last $0.3 \mathrm{~cm}$ from the end of the range, gives a much closer fit with the formula holding for a single group, indicating that here the ratio between the mean values for the two groups of tracks is not far from one.

\footnotetext{
${ }^{4}$ Cf. e.g. N. Bohr, Phil. Mag. 30, 581 (1915).
} 


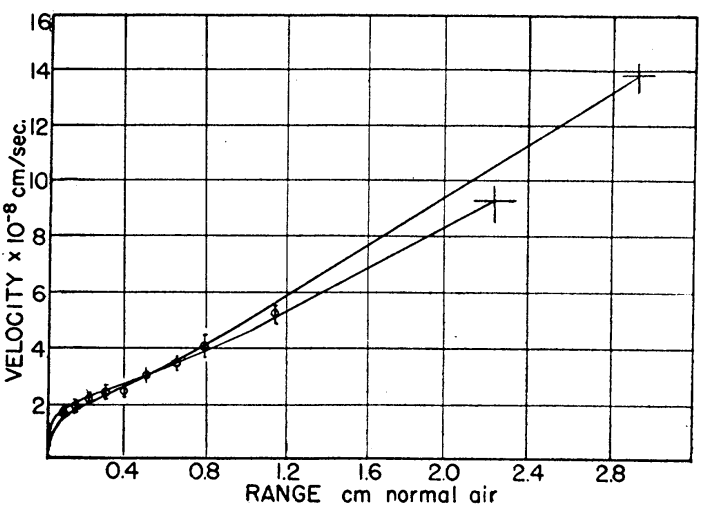

FIG. 1. Range-velocity relation for fission fragments.

Since the probability for energy transfer in nuclear collisions depends, apart from the velocity, simply on the charges and masses of the nuclei involved, the statistical analysis of the branch distribution along the tracks offers a direct means of obtaining the average rangevelocity relation for the fission fragments, provided the range-energy relation of the particles giving rise to the branches is known. Because of the large uncertainty of these relations for the low energies involved, and the difficulty of the identification of the branches in the photographs, the velocity determinations so obtained are not reliable in absolute measure, but their relative values give useful information about the general course of the range-velocity relation and they give a strong support for the conclusions previously drawn from direct measurements by individual large branches. ${ }^{1}$

The evidence regarding the range-velocity relation for the fission fragments derived from the material discussed is embodied in Fig. 1. The two curves correspond to the two main groups having different ranges and different initial velocities. These velocities are calculated assuming a mass ratio of $2: 3$ for the two nuclear fragments ejected with a total kinetic energy of $160 \mathrm{Mev} .{ }^{5}$ The attribution of the shorter range

\footnotetext{
${ }^{5}$ Because of an arithmetical error in the estimate of the average initial velocities of the fragments, the curve of
}

to the heavier particle follows directly from the general character of the curves for the higher velocities, where the course is nearly linear and the slopes should not differ greatly for the two groups of fragments. The shape of the curves for the lower velocities is determined from the branch counts. The points indicated in the figure give on a suitable scale the mean velocities in this region, derived from the number of branches within given limits of length in various intervals of the range.

Since the steep descent at the end of the range must be ascribed to the effect of numerous nuclear collisions, ${ }^{2}$ the velocity of the heavy fragment should be the higher in this region, as is also indicated from the study of the branch distribution. In fact, the probability of branching due to nuclear collision is proportional to $\left(Z^{2} / V^{2}\right)$, where $Z$ is the charge number and $V$ is the velocity; the close equality of the average number of branches for the two groups in this region therefore implies that the effect of the charge difference is partially neutralized by the difference in velocity. On the other hand, the large difference for the groups in the average number of branches at greater distances from the end of the range is a clear indication that here the velocity of the lighter fragment is the greater. In this region, the rate of decrease of momentum is approximately the same for the two types of fragments as would be expected from the general theoretical arguments. Still, this rate appears to be somewhat larger for the heavier particle, pointing to a slightly higher net charge for the same velocity, as is also consistent with the looser binding of the outer electrons in an atom of greater atomic number.

The various points discussed in this note will be more closely considered in the two forthcoming papers in the Communications of the Copenhagen Academy to which reference has already been made in our earlier articles.

Fig. 3 in the earlier note was drawn too high. This correction does not, however, alter the conclusions regarding the general character of the curve. 